\title{
OR7-004 - Validation of AIDAI score
}

\author{
M Piram ${ }^{1 *}$, I Koné-Paut ${ }^{1}$, M Gattorno ${ }^{2}$, N Ruperto ${ }^{2}$, the AIDAI Validation Study Group \\ From 7th Congress of International Society of Systemic Auto-Inflammatory Diseases (ISSAID) \\ Lausanne, Switerland. 22-26 May 2013
}

\section{Introduction}

With the increasing potential for targeted therapies in autoinflammatory diseases, there is the need for validated and standardized assessment tools which can be used to evaluate the level of disease activity and response to therapy.An international collaboration, initiated by Assistance Publique-Hôpitaux de Paris (APHP) in association with the Paediatric Rheumatology International Trials Organization (PRINTO at www.printo.it) and supported by the EUROFEVER and EUROTRAPS networks, has previously designed the content and the preliminary scoring of an Auto-Inflammatory Disease Activity Index (AIDAI).

\section{Objectives}

To validate the AIDAI score in the four major hereditary recurrent fever syndromes (HRFs): familial Mediterranean fever (FMF), mevalonate kinase deficiency (MKD), tumor necrosis factor receptor-associated periodic syndrome (TRAPS), and cryopyrin-associated periodic syndromes (CAPS).

\section{Methods}

In 2010, an international collaboration established the content of a disease activity tool for HRFs. Patients completed a one-month prospective diary with 12 yes/no (dichotomous) items prior to a clinical appointment during which their physician assessed their disease activity by a questionnaire. Eight international experts in autoinflammatory diseases evaluated patient's disease activity by a blinded web-evaluation and a nominal group technique consensus conference with their consensus judgment considered as gold standard. Sensitivity/specificity/ accuracy measures and the ability of the score to discriminate active versus inactive patients via the best cut-off score were calculated by a receiver operating characteristic (ROC) analysis.

\section{Results}

Consensus was achieved for 98/106 (92\%) cases (39 FMF, 35 CAPS, 14 TRAPS and 10 MKD) with 26 patients declared as having inactive disease and 72 active disease. The median total AIDAI score was 14 (range $=0-175$ ). An AIDAI cut-off score $\geq 9$ discriminated active versus inactive patients, with sensitivity/specificity/accuracy of $89 \% / 92 \% / 90 \%$ respectively and an area under the curve of $98 \%(95 \% \mathrm{CI}=96 \%>100 \%)$.

\section{Conclusion}

The AIDAI score is a valid and simple tool for the assessment of disease activity in FMF/MKD/ TRAPS/ CAPS This tool is easy to use in clinical practice and has the potential to be used as a standard efficacy measure in future clinical trials.

\section{Disclosure of interest}

None declared.

Authors' details

${ }^{1}$ Pediatrics and Pediatric Rheumatology, $\mathrm{CHU}$ de Bicêtre, University of Paris Sud, Le Kremlin Bicêtre, France. ²Pediatria II, Reumatologia, Istituto G Gaslini, PRINTO Coordinating Centre, Genova, Italy.

Published: 8 November 2013

doi:10.1186/1546-0096-11-S1-A105

Cite this article as: Piram et al:: OR7-004 - Validation of AIDAI score. Pediatric Rheumatology 2013 11(Suppl 1):A105. 